\title{
Clinical and therapeutic implications of psychiatric comorbidity in high functioning autism/Asperger syndrome: An Italian study
}

\author{
Silvia Giovinazzo ${ }^{1}$, Sara Marciano ${ }^{1}$, Grazia Giana ${ }^{2}$, Paolo Curatolo ${ }^{1}$, Maria-Cristina Porfirio ${ }^{1}$ \\ ${ }^{1}$ Department of Child Neurology and Psychiatry, Tor Vergata University, Rome, Italy \\ ${ }^{2}$ Department of Neuroscience, Child and Adolescence Psychiatry Unit, Children Hospital Bambino Gesù, Rome, Italy \\ Email: silviagiovinazzo@yahoo.it
}

Received 10 March 2013; revised 12 April 2013; accepted 20 April 2013

Copyright (C 2013 Silvia Giovinazzo et al. This is an open access article distributed under the Creative Commons Attribution License, which permits unrestricted use, distribution, and reproduction in any medium, provided the original work is properly cited.

\begin{abstract}
The present study describes the occurrence of psychiatric comorbid disorders in a cohort of 86 high functioning autism (HFA)/Asperger syndrome (AS) patients, examined at Child Neurology and Psychiatry Unit of Tor Vergata University. 38 patients out of 86 $\mathbf{( 4 4 . 2 \% )}$ presented one or more psychiatric comorbidities, such as mood disorders, Attention Deficit/Hyperactivity Disorder (ADHD), Tourette syndrome (TS), anxiety disorders, including obsessive-compulsive disorder (OCD), and psychotic symptoms. We compared our sample with the evidences from the scientific literature on psychiatric comorbidity in ASD patient, in particular in HFA/AS. In this paper we focus on the high frequency of comorbid psychiatric disorders in HFA/AS patients, such as mood disorders, Attention Deficit/Hyperactivity Disorder (ADHD), Tourette syndrome (TS), anxiety disorders, including obsessive-compulsive disorder (OCD), and psychosis, including schizophrenia. We analyzed rates of all psichiatric comorbidities diagnosed in a sample of HFA/AS subjects and we compared findings from our study with the evidences from the scientific literature on psychiatric comorbidity in ASD patients, in particular HFA/AS. We point out that comorbid psychiatric symptoms can be hardly diagnosed, because they could present atipically in ASDs then in general population. Furthermore, they could be masked by ASD core symptoms.
\end{abstract}

Keywords: Autistic Spectrum Disorders; Psychiatric Comorbidity

\section{BACKGROUNDS}

Despite of the increased knowledge about the pathoge- netic mechanisms and an improvement of the diagnostic criteria, clinical practitioners have a really poor understanding that Autism Spectrum Disorders (ASDs) occur most frequently than thought in general population (prevalence rate for all ASD is estimated at 1\%) [1]. ASDs are neurobiological disorders, caused by complex gene-environment interactions, not yet fully identified [2].

Several studies on patients with ASDs indicate that up to $70.8 \%$ of them are affected by one or more psychiatric condition in comorbidity [3]. Psychiatric comorbidity occurs highly in patients with High Functioning Autism (HFA) and Asperger syndrome (AS) [4-6], which are milder forms of autism, according to some authors. HFA individuals have a good cognitive level, expressed by an IQ $>70$. AS is characterized by high verbal IQ, impaired social interaction, restricted interests and ritualistic behaviours, in absence of language delay.

It is still debated if a comorbid disorder could occur in ASD patients with a range of different symptoms from those ones that are pathognomonic in general population. The identification of psychiatric comorbidity is difficult in ASD patients: any psychiatric symptoms could overlap with or be masked by ASD symptoms themselves.

In this regard, HFA/AS patients could provide clinical information about their emotional states, due to their high cognitive level and great verbal communication. Moreover, those characteristics allow them to attend mainstream school and so they often experience more bullying and less social support, which seem to be related to increasing psychiatric comorbidities, like conduct problems and disruptive behaviors [1].

On the other hand, it was found that ASDs are much less recognized and diagnosed in those with milder core symptoms. HFA/AS patients may therefore present to health services for other psychiatric symptoms and these 
surface features may not clearly signal the presence of an ASD [1].

According to DSM-IV-TR [7], ADHD, OCD, schizophrenia are excluded from ASD diagnostic criteria and diagnosis of anxiety disorders is to be avoided. But many people with ASD also have a range of psychiatric symptoms quite corresponding to diagnostic criteria for those psychiatric disorders.

Furthermore, according new DSM-V diagnostic criteria, AS will not be provided for in ASDs. Nevertheless, we led our study while DSM-IV-TR criteria were still valid and we could recruit AS patients. However, both our and the other findings about psychiatric comorbidity in AS, hereafter should alert clinicians about the high risk of psychopathology in an "AS-like" people, although it can no longer be considered as "autistic" people.

Have not yet been identified specific risk factors for the development of co-morbidity in ASDs. Risk factors that are well recognized in general population to be associated with child psychiatric disorders show similar relationship in children with ASDs [1]. It has been demostrated that individuals with less severe ASDs, as HFA and AS, are equally likely to show additional psychiatric disorders [1].

Frequently are diagnosed anxiety and depression, behavioral disorders like ADHD or oppositional defiant disorder (ODD) and obsessive disorders [2,8,9]. Some studies report rare cases of comorbidity with schizophrenia and other disorders, including Tourette's syndrome, tics, trichotillomania, enuresis or encopresis. Many patients present multiple comorbid disorders: respectively $80 \%$ of ASDs plus ADHD, $60 \%$ of ASDs plus behavioral disorder, $40 \%$ of ASDs plus affective disorder have another or more comorbidities [3].

\section{CLINICAL STUDY}

\subsection{Objectives}

Our clinical study aimed to:

- Describe the psychiatric comorbid disorders in a cohort of HFA/AS patients;

- Define the prevalence of every psychiatric conditions in the sample, comparing our data with the literature;

- Identify how many comorbid psychiatric conditions have arisen in each patient;

- Identify a possible relationship between age of patients and the development of a specific comorbidity;

- Assess the drugs used for the treatment of psychiatric symptoms, defining how many patients needed to set up a polytherapy.

\subsection{Methods and Materials}

The sample consisted of 86 HFA/AS patients, examined at Child Neurology and Psychiatry Unit of Tor Vergata University, in Rome, from 2009 to 2012.

The patient's mean age was 14.9 years (median 13.1, SD 6.7), with a mean age at evaluation of 11.9 years (SD 7.1).

Male patients were 77 out of 86 (89\%), with a male/ female ratio of 8.5 .

Autistic symptoms were assessed by comparing the core clinical features with the DSM-IV diagnostic criteria for ASDs; furthermore, the diagnosis of ASD was supported by the Autism Diagnostic Observation Schedule (ADOS; Lord et al., 2000) and the Autism Diagnostic Interview-Revised (ADI-R; Lord et al., 1995).

Cognitive assessment was performed by the Wechsler Intelligence Scales (WISC-III; Wechsler, 1991) and non verbal Leiter International Performance Scale-Revised (Leiter-R-Visualization and Reasoning, Roid \& Miller, 1997).

The presence of psychiatric disorders in comorbidity was evaluated by:

- Kiddie-Schedule for Affective Disorders and Schizophrenia (K-SADS; Kaufman et al., 1997), a semistructured interview designed to assess present and past episodes of psychiatric disorders in children and adolescents between the ages of 6 - 18, according to DSM-IV criteria;

- Child Behavior Checklist (CBCL) 4/18 years, a questionnaire completed by parents, about children/adolescent behavioral (e.g. internalizing problems-withdrawal, somatic complaints, anxiety/depression-, delinquent behavior and externalizing behavior aggressive and also attention difficulties, social problems and thought problems);

- Conners’ Parent and Conners’ Teacher Rating Scale, which are questionnaires filled by parents or teachers, which provide to assess and quantify oppositional behavior, inattention, hyperactivity and ADHD Index;

- Swanson, Nolan, and Pelham-IV (SNAP-IV), a questionnaire designed to assist in diagnosing a child's behavioral problems, like Attention Deficit Disorder with Hyperactivity (ADHD) and Oppositional Defiant Disorder;

- Children's Depression Inventory (CDI), a brief selfreport test that assesses signs of depression in children and adolescents 7 to 17 years old;

- Multidimensional Anxiety Scale for Children (MASC), a self-report test that assesses the presence of symptoms related to anxiety disorders in youth aged 8 to 19 years.

\subsection{Results}

In our sample, all 86 subjects were high functioning, with full scale IQ > 70. Mean IQ was 92.4 (SD = 20.9). 
38 patients out of 86 (44.2\%) had one or more psychiatric comorbidities.

Attention deficit/Hyperactivity Disorder (ADHD) was the most common comorbidity, diagnosed in 25 patients (66\%).

Were also diagnosed bipolar disorder $(n=7 ; 18.4 \%)$, depression $(n=2 ; 5.3 \%)$, generalized anxiety disorder (GAD) $(n=2 ; 5.3 \%)$, positive psychotic symptoms $(n=$ 6 ; $15.8 \%)$, learning disorder $(n=1 ; 2.6 \%)$, obsessivecompulsive disorder ( $n=1 ; 2.6 \%)$, eating disorders $(n=$ $1 ; 2.6 \%)$, oppositional defiant disorder $(n=2 ; 5.3 \%)$, tics and Tourette's syndrome ( $n=2 ; 5.3 \%)$ (Table 1).

These 38 patients was divided in 3 groups: 1 ) only with one comorbidity (71\%), 2) with 2 comorbidity (24\%), 39 with 3 or more (5\%) (Table 2).

The association between ADHD and Bipolar Disorder occurred most frequently in patients with multiple comorbidities (36.4\%).

We studied the distribution of comorbidities within the sample (Table 3), according to four age ranges:

- Childhood, including 6- to 8-year and 11 months-old: $n=7$ (18.4\%);

- Pre-puberty: including 9- to 13-year and 11 monthsold subjects: $n=9(23.7 \%)$;

- Adolescence, including 14- to 17-year and 11 monthsold subjects: $n=11$ (28.9\%);

- Adulthood, including 18 years-old subjects and over: $n=11$ (28.9\%).

There was a higher incidence of comorbidity in adolescence. The prevalence of multiple comorbidities in-

Table 1. Comorbid disorders diagnosed in our sample.

\begin{tabular}{cc}
\hline Comorbid Disorder & Rate of patients (\%) \\
\hline Attention deficit/Hyperactivity disorder & 66 \\
Bipolar disorder & 18.4 \\
Psychosis & 15.8 \\
Depression & 5.3 \\
Tics and Tourette's syndrome & 5.3 \\
Generalized anxiety disorder & 5.3 \\
Oppositional defiant disorder & 5.3 \\
Eating disorder & 2.6 \\
Obsessive/Compulsive disorder & 2.6 \\
Learning disorder & 2.6 \\
\hline
\end{tabular}

Table 2. Rate of patients presenting one or more psychiatric comorbidities.

\begin{tabular}{cc}
\hline Comorbid psychiatric disorder & Rate of patients (\%) \\
\hline Any psychiatric disorder & $44.2 \%$ \\
One psychiatric disorder & $71 \%$ \\
Two psychiatric disorder & $24 \%$ \\
Three or more psychiatric disorder & $5 \%$ \\
\hline
\end{tabular}

Table 3. Distribution of comorbidities within our sample, according to age ranges.

\begin{tabular}{|c|c|c|c|c|}
\hline \multirow{3}{*}{ Comorbidity } & \multicolumn{4}{|c|}{ Age ranges } \\
\hline & \multicolumn{4}{|c|}{ [6,9] ys $[9,14]$ ys $[14,18]$ ys $>18$ ys } \\
\hline & $18.4 \%$ & $23.7 \%$ & $28.9 \%$ & $28.9 \%$ \\
\hline $\begin{array}{c}\text { Attention deficit/Hyperactivity } \\
\text { disorder }\end{array}$ & $\mathrm{X}$ & $\mathrm{X}$ & $\mathrm{X}$ & $\mathrm{X}$ \\
\hline Bipolar disorder & & $\mathrm{X}$ & $\mathrm{X}$ & $\mathrm{X}$ \\
\hline Psychosis & & & $\mathrm{X}$ & $\mathrm{X}$ \\
\hline Depression & & & & $\mathrm{X}$ \\
\hline Tics and Tourette’s syndrome & $\mathrm{X}$ & $\mathrm{X}$ & & \\
\hline Generalized anxiety disorder & & $\mathrm{X}$ & $\mathrm{X}$ & $\mathrm{X}$ \\
\hline Oppositional defiant disorder & & $\mathrm{X}$ & $\mathrm{X}$ & \\
\hline Eating disorder & & & & $\mathrm{X}$ \\
\hline Obsessive/Compulsive disorder & & & $\mathrm{X}$ & \\
\hline Learning disorder & & & & $\mathrm{X}$ \\
\hline
\end{tabular}

creased in puberty and in adolescence.

Every age range was characterized by suffering from specific types of comorbidity.

Of the 38 patients with comorbidities, 19 (50\%) received pharmacological treatment. Regarding disruptive behaviours, most of our ASD plus ADHD patients responded to $\mathrm{MPH}$, one patient did not tolerate that drug and had to change with atomoxetine, and another patient was treated only with atomoxetine. The patients with ODD also were treated with methylphenidate.

Psychotic symptoms were treated with atyical antipychotics, as well as olanzapine or risperidone in most cases, or aripiprazole in a single case.

The patient with GAD received treatment with paroxetine (SSRI) and OCD was treated with sertraline (SSRI).

The patients with depressive disorder received benzodiazepines or venlafaxine (NSRI) and that with BD received Valproic Acid, which is the first line treatment for $\mathrm{BD}$ in youth. The case of eating disorders was treated with mirtazapine (NARI). $74 \%$ of the cases received a polypharmacotherapy.

\section{DISCUSSION}

We compared findings from our study with the evidences from the scientific literature on psychiatric comorbidity in individuals with ASD. We consider at first the only population-study dealing with several psychiatric comorbidities in ASD subject, involving a population-derived sample.

We found that $44.2 \%$ of our HFA/AS patients had comorbid psychiatric disorders. Our results differ from the study of Simonoff et al. [3]: they found that $70.8 \%$ of 112 ASD patients had at least one psychiatric comorbidity. However, that sample also included patients with 
low-functioning autism, which are more frequently and severely affected by psychiatric symptoms, as well as disruptive or repetitive behaviours, aggression, irritability [5]. Moreover, they employed more effective diagnostic tools for screening and diagnosis of psychiatric disorders in ASD, like Child and Adolescent Psychiatric Assessment (CAPA; Angold and Costello, 2000).

In 2009, Mukkades et al. [4] pointed out a $>90 \%$ prevalence of comorbidity in AS and HFA samples: the high rate was probably due to referral bias in diagnostic process.

In 2010, Mattila et al. [5] conducted a study on combined community- and clinic-sample of HFA/AS patients, aged from 9.8 to 16.3 years. They highlighted a comorbidity prevalence of $74 \%$, in a cohort aged more restrictely than ours. We considered also the age correlated with the comorbidity. This correlation is low and this aspect influenced the global prevalence in our population.

In our sample, $31 \%$ of patients had multiple comorbidities, as compared to 33\% reported by Simonoff et al. [3], and to $37 \%$ by Mattila et al. [5]. The same comorbidities have been identified in these studies, as well as in ours: ADHD, GAD, OCD, ODD, eating behavior disorders, $\mathrm{BD}$ and depression, tics and TS, learning disorders and psychosis. This recurrent finding of the same associations between disorders emphasizes that they have a common genetic and neurobiological substrate. In our sample, ADHD is the most frequent comorbid disorder (66\%), specially presenting in childhood and puberty. In literature, the prevalence of ADHD among individuals with ASD is estimated to be $28.2 \%$ [3], particularly among those with HFA/AS it's $44 \%$ - 65\% [6]. These findings stress the clinical necessity to assess inattention and hyperactivity/impulsivity in ASD [10], and point out also the fact that often is difficult to establish early diagnosis of HFA/AS, because autistic symptoms could be masked by ADHD symptoms.

The neurobiological point of view shows that ASD and ADHD are deficits in executive functioning and reflect a common dysfunction of fronto-temporal circuits [11]. ASD and ADHD also share genetic background: some chromosomal regions such as $2 \mathrm{q} 24,16 \mathrm{p} 13,16 \mathrm{p} 1$, $17 \mathrm{p} 11,5 \mathrm{p} 13,15 \mathrm{q}$ have been identified as susceptibility loci to both ADHD and ASD [12].

Furthermore, two patients had oppositional defiant disorder (5.6\%), compared to $1 \%$ in the study of Simonoff, to $16 \%$ according to Mattila et al. [5], up to $30 \%$ according Mukkades et al. [4]. Must be considered that aggression, often found in ODD, is common in ASD patients, most of all in LFA; moreover, could be the epiphenomenon of other types of comorbidity, e.g. mood disorders or anxiety [5].

Bipolar disorder occurred in $5.2 \%$ of cases, specially in adolescence, as well as other mood disorders. These data are in accord to the literature [13-16] (prevalence of $2 \%$ - 9\%, usually in adolescence).

Depression occurred in 5.2\%, with onset in adulthood, compared with $0.9 \%$ evidenced by Simonoff [3]. Depression can often arise with an increase of withdrawal and aggressive behaviours: these symptoms may present more clearly in patients with HFA/AS, rather than in low-functioning autism (LFA), which could primarily presenting withdrawal and aggression. From other studies has been reported a very broad range of prevalence of mood disorders, from $6 \%$ up to $70 \%[4,5,15-19]$. This variability could depend to the fact that some authors examined only AS patients, other ones considered depressive symptoms rather then a diagnosis of major depression.

Only one adolescent patient presented OCD and two individuals had a GAD (5.26\%), one at puberty, the other at about 18 years. Simonoff et al. [3] found that $2 \%$ of ASD patients developed a OCD and up to $44 \%$ an anxiety disorders. According to the literature, OCD is the anxiety disorder that appears most frequently in ASD $[3,20,21]$.

It is possible to recognize obsessions/compulsions from ASD repetitive behaviours, [6], but standardized diagnostic tools are currently not yet available.

In our sample, $15.8 \%$ of patients presented psychotic symptoms, with onset in adulthood. In literature, the prevalence of schizophrenia in ASD is of $0.6 \%$ [11], in HFA/AS up to $3.3 \%$ [4]. In adults with schizophrenia, there is often a history of autistic symptoms in childhood; autism and schizophrenia share some neurobiological abnormalities and genetic backgrounds $[17,18]$.

According to all literature examined [3], tics and Tourette's syndrome had an overall prevalence of $22 \%$ $30 \%[4,5,6,22]$.

Indeed, TS is considered a risk factor for development of further neuropsychiatric disorder, in ASD subjects [22, 23]. In our sample, two individuals presented tics only as comorbidity.

About pharmacoterapy in ASDs [24], atypical antipsychotics or neuroleptics can be effective against aggression and core symptoms; but it is often necessary to administer multiple drugs to control comorbid psychiatric symptoms. Methylphenidate is the first choice for the treatment of ADHD, benzodiazepines for anxiety symptoms, SSRI's for the treatment of OCD and mood disorders.

This study has some limitations, at first by having a moderate sample size, a too wide age range and no comparison group. The age-ranged groups were not wellmatched in gender and number of patients. Second, the assessment by the parents and children/adolescents may have resulted in some recall and/or referral bias. Furthermore, we have not distinctly considered current psy- 
chiatric diagnosis from past comorbidity.

Third, the present study only provides the rate and type of psychiatric disorders, and there was no information provided regarding associated risk factors such as family history and life events.

In addition, concerning the diagnostic instruments used in this study, there are no algorithms precisely for ASD.

Regarding to the pharmacological treatment, it would be necessary to involve and monitorize a larger sample of treated patients, to verify its short and long term efficacy.

\section{CONCLUSIONS AND FUTURE PROSPECTIVES}

There is still no consensus about the belief that symptoms of new onset in ASD should be classified as a manifestation of further psychiatric disorder.

It could be useful to assess the possible common pathogenetic mechanisms and risk factors between ASDs and other psychiatric disorders, and to obtain additional information on the underlying disfunction [25].

In literature there are only few data about comorbid psychiatric disorders in ASDs. It could be necessary to increase the sample size and to collect the largest number of clinical-anamnestic information, for studying etiopathogenesis and risk factors for the occurrence of comorbidity [26,27]; meta-analysis studies would be useful, to compare individual researches. It would be interesting to highlight a possible correspondence between age and the development of a specific comorbid disease. This report may be useful for lifetime monitoring patients, to predict the onset of a specific disease, and for early recognition of symptoms, to plan a focused and effective treatment.

According to some authors, there is need for caution in interpreting results that use generic measures in higlhy specific and distinct populations, as well as ASDs, without first characterizing the instrument properties, e.g. scoring and cut-offs, pertaining to that population [1].

Clinical practicioners should be able to use diagnostic tools which are specific for the diagnosis of comorbidity in ASD patients, to recognize atypical or masked symptoms (e.g. Autism Comorbidity Interview Present and Lifetime version; ACI-PL; Lefeyer et al., 2006; Developmental Disability Child Global Assessment Scale; DDCGAS; Wagner et al., 2007). It would also be necessary to update the diagnostic criteria for psychiatric disorders most commonly associated with ASD, adapting them to the characteristics of these patients.

\section{REFERENCES}

[1] Simonoff, E., Jones, C.R., Baird, G., Pickles, A., Happé, F. and Charman, T. (2013) The persistence and stability of psychiatric problems in adolescents with autism spectrum disorders. Journal of Child Psychology and Psychiatry, 54, 186-194. doi:10.1111/j.1469-7610.2012.02606.x

[2] Abrahams, B.S. and Geschwind, D.H. (2008) Advances in autism genetics: On the threshold of a new neurobiology. Nature Genetics, 9, 341-353.

[3] Simonoff, E., Pickles, A., Charman, T., Chandler, S., Loucas, T. and Baird, G. (2008) Psychiatric disorders in children with autism spectrum disorders: Prevalence, comorbidity, and associated factors in a population-derived sample. Journal of the American Academy of Child and Adolescent Psychiatry, 47, 921-929.

doi:10.1097/CHI.0b013e318179964f

[4] Mukaddes, N.M., Hergüner S. and Tanidir, C. (2010) Psychiatric disorders in individuals with high-functioning autism and Asperger's disorder: Similarities and differences. World Journal of Biological Psychiatry, 11, 964971. doi:10.3109/15622975.2010.507785

[5] Mattila, M.L., Hurtig, T., Haapsamo, H., et al. (2010) Comorbid psychiatric disorders associated with Asperger syndrome/high-functioning autism: A community- and clinic-based study. Journal of Autism and Developmental Disorders, 40, 1080-1093. doi:10.1007/s10803-010-0958-2

[6] Mazzone, L., Ruta, L. and Reale, L. (2012) Psychiatric comorbidities in Asperger syndrome and high functioning autism: Diagnostic challenges. Annals of General Psychiatry, 11, 16.

[7] American Psychiatric Association (2000) Diagnostic and statical manual of mental disorders. 4th Edition, APA, Washington, DC.

[8] Kim, J.A., Szatmari, P., Bryson, S.E., Streiner, D.L. and Wilson, F. (2000) The prevalence of Anxiety and mood problems among children with autism and Asperger syndrome. Autism, 4, 117-132. doi:10.1177/1362361300004002002

[9] Gadow, K.D., DeVincent, C.J. and Schneider, J. (2009) Comparative study of children with ADHD only, autism spectrum disorder + ADHD, and chronic multiple tic disorder + ADHD. Journal of Attention Disorders, 12, 474-485. doi:10.1177/1087054708320404

[10] Yoshida, Y. and Uchiyama, T. (2004) The clinical necessity for assessing Attention Deficit/Hyperactivity Disorder (ADHD) symptoms in children with high-functioning Pervasive Developmental Disorder (PDD). European Child \& Adolescent Psychiatry, 13, 307-314. doi:10.1007/s00787-004-0391-1

[11] Sinzig, J., Morsch, D., Bruning, N., Schmidt, M.H. and Lehmkuhl, G. (2008) Inhibition, flexibility, working memory and planning in autism spectrum disorders with and without comorbid ADHD-symptoms. Child and Adolescent Psychiatry and Mental Health, 2, 4-15. doi:10.1186/1753-2000-2-4

[12] Rommelse N.N., Geurts H.M., Franke B., Buitelaar J.K., Hartman C.A. (2011) A review on cognitive and brain endophenotypes that may be common in autism spectrum disorder and attention-deficit/hyperactivity disorder and facilitate the search for pleiotropic genes. Neuroscience 
\& Biobehavioral Reviews, 35, 1363-1396. doi:10.1016/j.neubiorev.2011.02.015

[13] Raja, M. and Azzoni, A. (2008) Comorbidity of Asperger's syndrome and Bipolar disorder. Clinical Practice and Epidemiology in Mental Health, 4, 26-31. doi:10.1186/1745-0179-4-26

[14] Munesue, T., Ono, Y., Mutoh, K., Shimoda, K., Nakatani, H. and Kikuchi, M. (2008) High prevalence of bipolar disorder comorbidity in adolescents and young adults with high-functioning autism spectrum disorder: A preliminary study of 44 outpatients. Journal of Affective Disorders, 111, 170-175. doi:10.1016/j.jad.2008.02.015

[15] Hofvander, B., Delorme, R., Chaste, P., Nydén, A., Wentz, E., Ståhlberg, O., et al. (2009) Psychiatric and psychosocial problems in adults with normal-intelligence autism spectrum disorders. BMC Psychiatry, 10, 35.

[16] Lugnegård, T., Hallerbäck, M.U. and Gillberg, C. (2011) Psychiatric comorbidity in young adults with a clinical diagnosis of Asperger syndrome. Research in Developmental Disabilities, 32, 1910-1917. doi:10.1016/j.ridd.2011.03.025

[17] Rapoport, J., Chavez, A., Greenstein, D., Addington, A. and Gogtay, N. (2009) Autism-spectrum disorders and childhood onset schizophrenia: Clinical and biological contributions to a relationship revisited. Journal of the American Academy of Child and Adolescent Psychiatry, 48, 10-18. doi:10.1097/CHI.0b013e31818b1c63

[18] Stahlberg, O., Soderstrom, H., Rastam, M. and Gillberg, C. (2004) Bipolar disorder, schizophrenia, and other psychotic disorders in adults with childhood onset $\mathrm{AD} / \mathrm{hd}$ and/or autism spectrum disorders. Journal of Neural Transmission, 111, 891-902. doi:10.1007/s00702-004-0115-1

[19] Ghaziuddin, M., Ghaziuddin, N. and Greden, J. (2002) Depression in persons with autism: Implications for research and clinical care. Journal of Autism and Developmental Disorders, 32, 299-306. doi:10.1023/A:1016330802348
[20] Russel, A.J., Mataix-Cols, D., Anson, M. and Murphy, D.G.M. (2005) Obsessions and compulsions in Asperger syndrome and high-functioning autism. British Journal of Psychiatry, 186, 525-528. doi:10.1192/bjp.186.6.525

[21] Ruta, L., Mugno, R., Genitori, D’Arrigo, V., Vitiello, B. and Mazzone, L. (2008) Obsessive-compulsive traits in children and adolescents with Asperger syndrome. European Child \& Adolescent Psychiatry, 19, 17-24. doi:10.1007/s00787-009-0035-6

[22] Burd, L., Li, Q., Kerbeshian, J., Klug, M. G. and Freeman, R.D. (2009) Tourette syndrome and comorbid pervasive developmental disorders. Journal of Child Neurology, 24 170-175. doi:10.1177/0883073808322666

[23] Canitano, R. and Vivanti, G. (2007) Tics and Tourette syndrome in autism spectrum disorders. Autism, 11, 1928. doi:10.1177/1362361307070992

[24] Benvenuto, A., Battan, B., Porfirio, M.C. and Curatolo, P. (2013) Pharmacotherapy of autism spectrum disorders. Brain \& Development, 35, 119-127. doi:10.1016/j.braindev.2012.03.015

[25] Gadow, K.D., Roohi, J., DeVincent, C.J. and Hacthwell, E. (2008) Association of ADHD, tics, and anxiety with dopamine transporter (DAT1) genotype in autism spectrum disorder. Journal of Child Psychology and Psychiatry, 49, 1331-1338. doi:10.1111/j.1469-7610.2008.01952.x

[26] Kanne, S.M., Abbacchi, A.M. and Constantino, J.N. (2009) Multi-informant ratings of psychiatric symptom severity in children with autism spectrum disorders: The importance of environmental context. Journal of Autism and Developmental Disorders, 39, 856-864. doi:10.1007/s10803-009-0694-7

[27] Hurtig, T., Kuusikko, S., Mattila, M.L., et al. (2009) Multi-informant reports of psychiatric symptoms among high-functioning adolescents with Asperger syndrome or autism. Autism, 13, 583-598. doi:10.1177/1362361309335719 